\title{
Top 20 translational researchers in 2013
}

\author{
Brady Huggett \& Kathryn Paisner
}

We present a ranking of researchers based on paper and patent output. Patent analytics firm IP Checkups counted patents assigned to faculty members at their current affiliations who were corresponding authors on the $\sim 200$ research papers highlighted in 2013 in SciBX, a weekly publication that scans 50 select journals for articles of translational interest.
The rankings, of the top senior (Table 1) and junior faculty (Table 2), are biased to favor researchers who have been at their current affiliation for an extended period. Figure 1 shows the area of research focus for papers highlighted in SciBX in 2013. Cancer dominated at nearly $30 \%$ of the highlighted papers; other areas of interest included fibrosis and the microbiome.

Table 1 Top translational researchers in 2013, ranked by total patents at their current affiliation.

\begin{tabular}{|c|c|c|c|c|}
\hline \multirow[t]{2}{*}{ Professor, affiliation } & \multirow[t]{2}{*}{ SciBx paper(s) } & \multicolumn{3}{|c|}{ Patents } \\
\hline & & US & Europe & Total \\
\hline Peter G. Schultz, The Scripps Research Institute, La Jolla, Calif. & $\begin{array}{l}\text { Deshmukh, V.A. et al. Nature 502, 327-332 (2013). } \\
\text {-Wang, F. et al. Proc. Natl. Acad. Sci. USA 110, E2510-E2517 (2013). }\end{array}$ & 81 & 12 & 93 \\
\hline $\begin{array}{l}\text { James M. Wilson, Perelman School of Medicine at the University of Pennsylvania, } \\
\text { Philadelphia }\end{array}$ & Limberis, M.P. et al. Sci. Transl. Med. 5, 187ra72 (2013). & 62 & 28 & 90 \\
\hline $\begin{array}{l}\text { Guillermo J. Tearney, Massachusetts General Hospital and Harvard Medical } \\
\text { School, Boston }\end{array}$ & Gora, M.J. et al. Nat. Med. 19, 238-240 (2013). & 67 & 16 & 83 \\
\hline Stuart L. Schreiber, Broad Institute of MIT and Harvard, Cambridge, Mass. & Basu, A. et al. Cell 154, 1151-1161 (2013). & 53 & 2 & 55 \\
\hline Mark E. Davis, California Institute of Technology, Pasadena, Calif. & Wiley, D.T. et al. Proc. Natl. Acad. Sci. USA 110, 8662-8667 (2013). & 44 & 5 & 49 \\
\hline Atsushi Miyawaki, RIKEN Brain Science Institute, Saitama, Japan & Kumagai, A. et al. Cell 153, 1602-1611 (2013). & 27 & 15 & 42 \\
\hline $\begin{array}{l}\text { William R. Jacobs Jr., Albert Einstein College of Medicine of Yeshiva University, } \\
\text { Bronx, N.Y. }\end{array}$ & Wang, F. et al. Proc. Natl. Acad. Sci. USA 110, E2510-E2517 (2013). & 37 & 2 & 39 \\
\hline Adolfo García-Sastre, Icahn School of Medicine at Mount Sinai, New York & Langlois, R.A. et al. Nat. Biotechnol. 31, 844-847 (2013). & 31 & 7 & 38 \\
\hline $\begin{array}{l}\text { Richard A. Young, Whitehead Institute for Biomedical Research, Cambridge, } \\
\text { Mass. }\end{array}$ & $\begin{array}{l}\text { Whyte, W.A. et al. Cell 153, 307-319 (2013). } \\
\text { •Lovén, J. et al. Cell 153, 320-334 (2013). }\end{array}$ & 30 & 7 & 37 \\
\hline Alexander Levitzki, The Hebrew University of Jerusalem, Jerusalem & Reuveni, H. et al. Cancer Res. 73, 4383-4394 (2013). & 22 & 9 & 31 \\
\hline Gerald B. Pier, Harvard Medical School and Brigham and Women's Hospital, Boston & Cywes-Bentley, C. et al. Proc. Natl. Acad. Sci. USA 110, E2209-E2218 (2013). & 26 & 4 & 30 \\
\hline David Baltimore, California Institute of Technology & Balazs, A.B. et al. Nat. Biotechnol. 31, 647-652 (2013). & 23 & 6 & 29 \\
\hline Rudolph E. Tanzi, Harvard Medical Schoola & Veeraraghavalu, K. et al. Science 340, 924 (2013). & 23 & 3 & 26 \\
\hline George M. Church, Harvard Medical School & Mali, P. et al. Science 339, 823-826 (2013). & 20 & 4 & 24 \\
\hline David A. Scheinberg, Memorial Sloan-Kettering Cancer Center, New York & Dao, T. et al. Sci. Transl. Med. 5, 176ra33 (2013). & 18 & 5 & 23 \\
\hline Arul M. Chinnaiyan, University of Michigan Medical School, Ann Arbor, Mich. & Velusamy, T. et al. Proc. Natl. Acad. Sci. USA 110, 3035-3040 (2013). & 15 & 8 & 23 \\
\hline George Georgiou, The University of Texas at Austin, Austin, Texas & DeKosky, B.J. et al. Nat. Biotechnol. 31, 166-169 (2013). & 18 & 4 & 22 \\
\hline $\begin{array}{l}\text { Ziad Mallat, Institut National de la Santé et de la Recherche Médicale } \\
\text { (INSERM), Paris }\end{array}$ & Zouggari, Y. et al. Nat. Med. 19, 1273-1280 (2013). & 13 & 9 & 22 \\
\hline Richard T. Lee, Brigham and Women's Hospital & Loffredo, F.S. et al. Cell 153, 828-839 (2013). & 16 & 2 & 18 \\
\hline Dean Sheppard, University of California at San Francisco School of Medic & Henderson, N.C. et al. Nat. Med. 19, 1617-1624 (2013). & 13 & 5 & 18 \\
\hline
\end{tabular}

San Francisco, Calif.

apatents assigned to General Hospital Corp.

Table 2 Top translational junior faculty in 2013, ranked by total patents at their current affiliation.

Professor, affiliation

SciBx paper(s)

Omid C. Farokhzad, Brigham and Women's Hospital, Boston

James E. Bradner, Dana-Farber Cancer Institute, Boston

Frank Alexis, Clemson University, Clemson, S.C.

Tarek M. Fahmy, Yale University, New Haven, Conn.

Yuan-Xiang Tao, The Johns Hopkins University School of Medicine, Baltimore

Pridgen, E.M. et al. Sci. Transl. Med. 5, 213 ra167 (2013).

Puissant, A. et al. Cancer Discov. 3, 308-323 (2013).

Pridgen, E.M. et al. Sci. Transl. Med. 5, 213ra167 (2013).

Look, M. et al. J. Clin. Invest. 123, 1741-1749 (2013).

Zhao, X. et al. Nat. Neurosci. 16, 1024-1031 (2013).

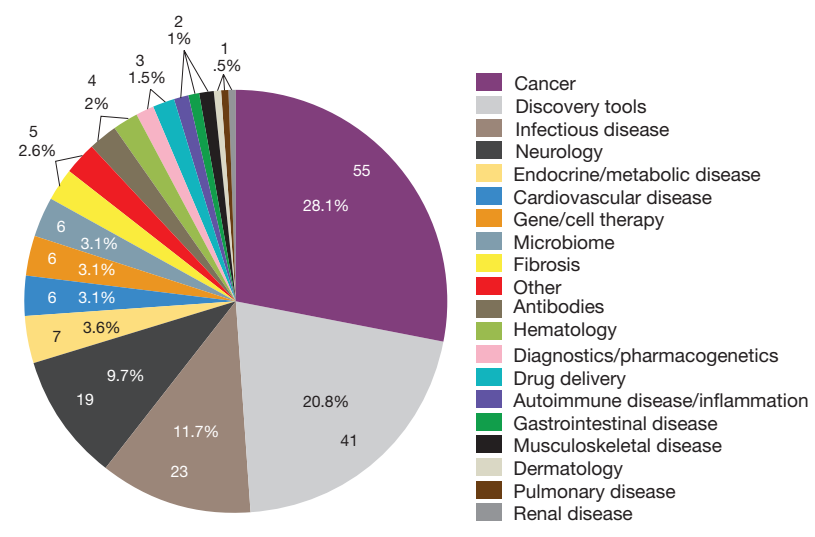

Figure 1 Areas of focus by percentage for research papers highlighted in SciBX in 2013.

\section{First Rounders Podcast: Julian Davies}

Julian Davies is a professor emeritus at the University of British Columbia. He was one of the founding fathers of biotech as the research director at Biogen's European lab in the early 1980s. Nature Biotechnology talked to Davies about his childhood in Wales during World War II, breakthroughs in antibiotic research and his life in the lab. http://www.nature.com/nbt/podcast/index.html.

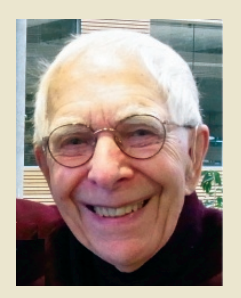

Brady Huggett is business editor at Nature Biotechnology. Kathryn Paisner is director of research and analytics at IP Checkups Inc., Berkeley, California,USA.e-mail:kpaisner@ipcheckups.com. 